Gmelinsche Reaktion. Die Löslichkeitsverhältnisse und das spektroskopische Verhalten des im alkoholischen Filtrate enthaltenen gelben Farbstoff entsprachen auch denen des Bilirubins. - Die Bilirubinkonzentration des Blutes betrug am Ende der Versuche $0,0005-0,002 \%$.

In der dritten Stunde des Versuches zeigte das in dem Milzhilus befindliche und ursprünglich blaß rosafarbene Fettgewebe eine blaßgelbe Verfärbung, welche Verfärbung sich beiläufig bis zum Ende der vierten Stunde steigerte, und da war das Fettgewebe schon ausgesprochen gelb ${ }^{1}$ ). Diese Verfärbung wird nach unserer Auffassung durch das aus dem zirkulierenden Blute im Fettgewebe deponierte Bilirubin bedingt. Wir konnten zwar durch histologische Untersuchungen weder im Fettgewebe, noch in dem Milzgewebe Gallenfarbstoff nachweisen, der Nachweis desselben stößt aber nach Minzowski und NAUNYN ${ }^{2}$ ) bei Anwesenheit von geringen Mengen auf Schwierigkeiten. Auch auf chemischem Wege vermochten wir im. Fettgewebe - Whipples und Hoopers ${ }^{3}$ ) etwas wortkarg beschriebenes Verfahren befolgend - kein Bilirubin nachzuweisen.

Nach Abschluß des Versuches untersuchten wir die Milz histologisch, hauptsächlich um zu ermitteln, wieviel und in welcher Form befindliches Eisen in ihr nachweisbar sei. Im allgemeinen fanden wir in der Milz nach dem Versuche ziemlich viel Hämosiderin. Um festzustellen, wieviel hiervon eventuell während des Versuches gebildetes Eisen sei, entfernten wir in einem Falle ein Stück der Milz vor dem Versuche mittels Thermokauters und kontrollierten auf diese Art den Eisengehalt der Milz vor und nach dem Versuche. Es ergab sich, daß die Milz bereits vor dem Versuche viel Hämosiderin enthielt, so $\mathrm{da} B$ wir unsere Untersuchnngen nach dieser Richtung noch nicht als abgeschlossen betrachten können.

Aus unseren Versuchen geht also hervor, daß unter den beschriebenen Umständen sich in der Milz Bilirubin bildet, und zwar in ziemlich ansehnlicher Menge. Das zur Durchströmung verwendete Blut enthielt am Ende der am besten gelungenen Versuche $2 \mathrm{mg}$ Bilirubin und es ist anzunehmen, daB im Milzgewebe selbst und in dem mit dem Hilus zusammenhängenden Fette vielleicht die Hälfte dieser Menge enthalten war, so daß wir die Menge des im Verlaufe von vier Stunden gebildeten Bilirubins beiläufig auf $3 \mathrm{mg}$ setzen können. Nach STADELMANN ${ }^{4}$ ) beträgt die Gallenpigmentproduktion des Hundes pro $\mathrm{kg}$ in 24 Stunden höchstens $7 \mathrm{mg}$. Somit erzeugt ein Hund von $17,5 \mathrm{~kg}$ (das durchschnittliche Gewicht der für unsere Versuche verwendeten Hunde) in 4 Stunden höchstens 20,4 mg Bilirubin. Unter den beschriebenen Versuchsbebingungen vermag daher die Milz den siebenten Teil der normalen Bilirubinproduktion hervorzubringen. Diese Fähigkeit der Milz kann beim lebenden Tiere vielleicht noch stärker sein. Wenn wir bedenken, daß außer der Milz auch ándere Organe hinsichtlich der extrahepatogenen Bilirubinbildung zur Sprache kommen, so in erster Reihe die übrigen Elemente des reticulo-endothelialen Apparates (Knochenmark), ja vielleicht sogar jede Endothelzelle (Whipples und Hoopers, Hijmans, LESCHKES Versuche) so können wir jene Bilirubinmenge, welche ohne Zutun der Leber entstehen kann, nicht für eine zu vernachlässigende geringe Menge setzen. Dieser Prozeß kann besonders bei Anaemia perniciosa, beim hämolytischen Ikterus und bei Tolyulendiamin-Vergiftung von Bedeutung sein, wobei nach EPpingers und CHARNAss'5) Untersuchungen der Zerfall der roten Blutkörperchen und die Gallenpigmentbildung das Mehrfache, zuweilen wohl auch das Fünffache des normalen betragen kann. Da in diesen Fällen die Gallenpigmentbildung sehr groß ist, erscheint es annehmbar, daß das Gallenfarbstoffausscheidungsvermögen der Leber mit ihr nicht Schritt halten kann und ein Teil des Gallenpigmentes unserer Ansicht nach jener Teil, welcher sich nicht in der Leber bildete, sondern nur mittels des Blutstromes derselben zu-

1) In zwei Versuchen war diese Verfärbung neben geringem Bilirubingehalte des Serums gleichfalls gering.

2) MINKOWSKI und NAUNYN: zit. nach EPPINGER-RANZI l. c. S. 55.

3) WHIPPLE und HOOPER: 1. c.

4) STADELMANN : Der Ikterus zit. nach HAMMARSTEN: Lehrbuch der Phys:ol.

Chem. Wiesbaden. I9r4. S. 4 I2.

5) EPPINGER und CHARNAS: Zeitschr, f. klin. Med. Igr3. geführt wird, nicht zur Ausscheidung gelangt, oder nur teilweise ausgeschieden wird. Hierdurch wird die Bilirubinkonzentration im Blute erhöht und es entsteht Ikterus. Dieser Ikterus wird selbst in dem Falle, wenn wir voraussetzen, daß der größere Teil des Gallenpigmentes sich auch diesmal in der Leber bildet - vom extrahepatogenen Gallenpigment erzeugt.

Zusammenfassung: Es wird nachgewiesen, daß die über lebende Hundemilz Gallenpigment bildet, wenn sie mit defibriniertem Blute, welches Hämoglobin gelöst enthält durchströmt wird. Vor dem Versuche ist im zur Durchströmung benützten Blute Gallenpigment nicht vorhanden, am Ende der ersten Stunde enthält es das Blut in Spuren, später jedoch immer reichlicher, so daß am Ende der vierten Stunde die Diazoreaktion, sowie die Reaktionen von HaMmaRsten und GMelin ein positives Resultat ergeben. Auch die übrigen Eigenschaften des entstandenen Pigmentes sind jenen des Bilirubins gleich. Das im Milzhilus befindliche blaßrosafarbene Fettgewebe zeigt zu Ende des Versuches eine ausgesprochen ikterische Verfärbung. Die Menge des auf diese Weise gebildeten Bilirubins kann ungefähr den 7 . Teil der durch die Leber eines Hundes von derselben Größe in derselben Zeit durchschnittlich ausgeschiedenen Bilirubinmenge erreichen.

Eine ausführliche Beschreibung unserer Experimente erscheint an anderem Orte.

\section{KLINISCHER UND EXPERIMENTELLER BEITRAG ZUR KRAMPFLÖSENDEN WIRKUNG DER PURINDERIVATE.}

\author{
Von
}

Dr. S. HIRsch, Oberarzt.

Aus dem Städtischen Krankenhause Sandhof (Direktor: Professor Dr. ALWENS), und dem Pharmakologischen Institut der Universität Frankfurt a. M. (Direktor: Geheimrat Professor Dr. ELLINGER).

Die Krampfbereitschaft des Organismus ist in gewissem Maße abhängig von der Altersdisposition. Während man früher dem jugendlichen Alter prädilektorische Bedeutung für die Entstehung spastischer Zustände zuschrieb, hat man neuerdings den Nachweis spastischer pathogenetischer Faktoren für eine ganze Reihe von Krankheitsbildern auch im höheren Lebensalter erbringen können. Neben zentralnervösen Schädigungen sind hier vor allem spastische Erscheinungen bei und infolge von Kreislauferkrankungen zu erwähnen, deren Wirkungsmechanismus jedoch noch vielfach ungeklärt ist. Das gilt ganz besonders für die Symptome eines Krankheitsbildes, dessen klinische und therapentische Würdigung geradezu im umgekehrten Verhältnis steht zu seiner starken Verbreitung unter den arbeitenden Volksschichten und das unter den verschiedensten Bezeichnungen als "Marasmus", Senium, Emphysem, Myodegeneratio cordis, neuerdings auch als „Hypertonie“ zur klinischen Beobachtung kommt. Meist steht eine Symptomentrias im Vordergrund, die aus einer arteriellen Hypertonie, . katarrhalischen bronchitischen Erscheinungen mit Emphysem und Dyspnöe und mehr oder weniger ausgeprägten Herzrhythmusstörungen zusammengesetzt ist; daneben bestehen oft leichte Knöchelödeme. Sehr mit Recht hat neuerdings EPPINGER ${ }^{1}$ ) darauf hingewiesen, da $\beta$ das einseitige Interesse, das in solchen Fällen von ,sog. Myodegeneratio cordis", auch in therapeutischer Hinsicht, dem Herzmotor meist zugewandt wird, durchaus. nicht angebracht ist. Bei dauernder. Beobachtung einer großen Anzahl solcher Kranker über längere Zeit hinaus fiel uns immer wieder die Massenhaftigkeit und Vielgestaltigkeit der vorgebrachten Klagen im Gegensatz zu dem oft sehr dürftigen objektiven Organbefunde auf. Es scheint berechtigt, eine Mehrzahl der Besschwerden auf "funktionelle“ Ursachen zurückzuführen, und zwar dürften neben Symptomen von Gefäßschwäche spastische Zustände in bestimmten Gefäßbezirken eine Rolle spielen. Diese Annahme wird cx juvantibus

1j „Uber die sogen. Myodegeneratio cordis.“" Therap. d. Gegenw. Ig2I, S. 8J. 
gestützt. Dem ärztlichen Vorgehen kommen die Wünsche der Patienten entgegen: vielfach besteht geradezu ein Hunger nach krampflösenden Mitteln, nach Anaesthetica und Narkotica. Eine überaus quälende Agrypnie, die ätiologisch meist dunkel ist, bildet oft die Brücke zur allgemeinen, im wesentlichen symptomatischen Therapie. Der Gewohnheit, solche Kranke frühzeitig lediglich mit Narkotica zu behandeln, steht eine gewisse Polypragmasie gegenüber, die auf jedes Symptom der Krankheit, die vielleicht am treffendsten als chronische Kreislaufinsuffizienz zu bezeichnen wäre, mit einem neuen Medikament reagiert. Die sogenannte ätiologische Therapie bleibt meist unter einseitiger Hervorkehrung der Herzsymptome auf die Digitalis beschränkt. Bei dem Bestreben, die verschiedenen Komponenten der chronischen Kreislaufinsuffizienz der alten Leute $\mathrm{zu}$ unterscheiden und auszuwerten, und das therapeutische Handeln den tatsächlichen Vorgängen entsprechend möglichst einheitlich zu gestalten, ergaben sich uns nun für die Anwendung der Körper der Puringruppe einige, wenn auch nicht neue, so doch bisher in der Praxis noch nicht genügend gewürdigte Gesichtspunkte.

Gegenüber der vielfachen Anwendung der Purinkörper als $H e r z$ - und Gefäßmittel wie als Diuretica hat die seit längerer Zeit bekannte Wirkung der Purinderivate auf die Atmungsorgane außerordentlich wenig Beachtung gefunden, und zwar sowohl von experimenteller wie von klinischer Seite aus. Die experimentelle Begründung beruht im wesentlichen auf einer Beobachtung $P$. TRENDELENBURG ${ }^{1}$ ) bei seinen Untersuchungen an der isolierten Bronchialmuskulatur, wonach Coffein. natr. salicyl. in größeren Dosen einen der Alkoholwirkung ähnlichen Effekt hat. Gleichzeitig und unabhängig von diesen Versuchen konnte $\mathrm{PAL}^{2}$ ) am decerebrierten Meerschweinchen die Aufhebung von Bronchospasmen durch Coffein feststellen. PAL führt diese Wirkung auf eine periphere Erregung gewisser Åste des Sympathicus durch das Coffein zurück.

Noch weiter ist die therapeutische Verwertung dieser Untersuchungen zurückgeblieben; sie beschränkt sich auf gelegentliche Empfehlungen ${ }^{3}$ ). RoMBERG ${ }^{4}$ ) sieht den Nutzen des Coffeins und Theobromins bei Herzkranken im wesentlichen in der allgemeinen Anregung der Nerven und vielleicht auch der Muskeln. Er wendet die Mittel vor allem gern bei initialen arteriosklerotischen Störungen mit und ohne Hochdruck an. Bei der Behandlung dyspnoischer $\mathrm{Zu}$ stände gibt RoMBERG die Purinkörper im wesentlichen als Analeptica. Eine gewisse Sonderstellung wird nur dem Diuretin beim Asthma renale zugewiesen.

Die Grenzen der Purinkörpertherapie in der klinischen Praxis dürften im wesentlichen durch Applikations- und Dosierungsschwierigkeiten gezogen sein, ihre Fortschritte sind an die Herstellung besser löslicher Verbindungen, die man neuerdings, besonders in Frankreich, durch Allylierung ${ }^{5}$ ) zo gewinnen sucht, gebunden. Die Schwerlöslichkeit der wirksamsten Derivate und die Nebenwirkungen auf die Verdauungsorgane, die den bisher in lösliche Form gebrachten Purinderivaten, wie dem Diuretin zukommt, haben die Empfehlung anderer Präparate zur Förderung der Diurese angeregt.

Im allgemeinen wendet man jedoch zur Ưberwindung bronchospastischer Zustände vornehmlich stark wirkende Alkaloide, wie Atropin, die Narkotica und Adrenalin mit Vorliebe an. Selbstverständlich bedeutete deshalb eine Darreichungsform von Purinkörpern, die auch die Verwertung ihrer bronchodilatatorischen Wirkung gestattete, in mehr als einer Hinsicht eine Erleichterung des therapeutischen Vorgehens. In gewissem Grade scheint diese Eigenschaft einem uns von der Fabrik chem.-pharm. Präparate Dr. R. und Dr. O. WEIL zur Verfügung gestellten Präparate zuzukommen, das in handlicher Form die gleichzeitige Darreichung von

1) A. Pathol, U. Pharmakol. rgı 69, S. ro6.

8) Deutsche med. Wochenschr. I9I2, Nr. 38

3) S'ehe z. B. CURSCHMANN, Münch. med. Wochenschr. Ig2I, S. 395.

4) "Krankheiten des Herzens und der BlutgefäBe", 3. Aufl.. Stuttgart Igar S. $383 \mathrm{ff}$. Hier auch ausführliche Literatur zur Theripie des Kreislaufs.

5) S. POUCHET, Gaz. des hồ. Igzo, S. Is8r; BENECH, Rav. mé̉. de l'est. Igzi, 5. 88, cit. nach Kongr. Zntrbl. f. inn. Məd. BA. XVI u. XVIII.
Theobromin und Theophyllin gestattet. ${ }^{1}$ ) Ob den Dimethylxanthinen einzeln dargereicht, die gleiche oder eine ähnliche Wirkung zukommt, habe ich klinisch noch nicht geprüft. Schon bei den ersten Versuchen mit diesem Präparat, das als Suppositorium gegeben wird, kam neben der allgemeinen zirkulatorischen Wirkung vor allem eine überraschende günstige Beeinflussung der Atmungsstörungen zur Geltung.

Der therapeutische Erfolg, den wir an einer großen Versuchsreihe nachweisen konnten, läßt sich am besten aus der Mitteilung einiger Krankengeschichtsauszüge ersehen:

Friedrich Bl. Rittmeister a. D. 52 Jahre alt, eingeliefert am 26. April I92 I. Leidet seit angeblich 2 Jahren an einem Lungenkatarrh und an Herzbeschwerden. Digitalis und eine Nauheimer Badekur waren erfolglos. Klagt über schwerste Atemnot, die ihm das Gehen unmöglich macht, hat seit Wochen nicht geschlafen. Nächtliche Asthmaanfälle.

Befund: stark reduzierter Ernährungszustand, graue Gesichtsfarbe. Starke Cyanose der sichtbaren Schleimhäute, insbesondere auch der Conjunktiven, der Lippen, Gesicht aufgedunsen. Knöchelödeme. Hochgradigste Dyspnoe. Rasselnde Atmung mit exspiratorischem Stridor. Hautvenenkranz entlang dem linken Rippenbogen. Brustkorb sehr starr. Tiefstand der unteren. Lungengrenzen, beiderseits, hinten I2. Brustwirbeldorn bis I. Lendenwirbeldorn, vorn oberer Rand der 7. Rippe. Atemgeräusch sehr leise. Uberall pfeifende und schnurrende Geräusche, besonders in der rechten Hilusgegend. Perkussionsschall zeigt über der ganzen Lunge tympanitischen Charakter. Das Herz ist stark überlagert. Röntgenologisch: Emphysemherz. Töne leise. An der Spitze präsystolisches Geräusch. Arhythmia perpetua. Leber ist palpabel, überragt den Rippenbogen querfingerbreit.

Diagnose: schwerste Kreislaufinsuffizienz mit Stauungsbronchitis und Emphysem und mit - bzw. infolge von - (?) Herzmuskelinsuffizienz.

Therapie: Da ein Krankheitsbericht bei der Einlieferung nicht vorlag, und wir über etwa voraufgegangene Digitalismedikation nicht unterrichtet waren, erhält der Patient am gleichen Abend Suppositorium "Spasmopurin.". - Die Wirlung war im höchsten Maße überraschend. Patient gibt an, zum erstenmal seit langer Zeit nachts geschlafen $z u$ haben; er fühle sich wie neugeboren. In der Tat sind die Stauungserscheinungen an den Schleimhäuten, die Schwellungen und der Katarrh der Bronchien bereits bedeutend zurückgegangen. Die Atmung ist viel ruhiger, die Arrhytmie des Pulses, der jedoch noch sehr beschleunigt ist, weniger stark ausgeprägt.

Verlauf: Der Patient erhält nun weiter 2mal täglich 1 Suppos. Spasmopurin, vom 3. Tage an, 3 mal täglich $1 / 2$ Tablette Verodigen. Die Stauungserscheinungen schwinden innerhalb 4 Tagen vol]. ständig, die Atemnot ist ganz beseitigt. Pulsverlangsamung als Ausdruck der Digitaliswirkung tritt erst am 5. Tage der Verodigengaben auf. Am I2. Tage der Behandlung kann der seit Wochen bettlägerige Patient das Bett verlassen. Der Patient kann nach 4 Wochen als beschränkt arbeitsfähig entlassen werden.

Lilly B., 5 jährige Direktrice, seit 3 Jahren an einem chronischen Gelenkleiden erkrankt. Seit I Jahr ans Bett gefesselt. In letzter Zeit hochgradige Anfälle von Atemnot. Ausgesprochenes Schwächegefühl.

Befund: Blasse, sehr gealtert aussehende Frau in stark reduziertem Kräftezustand. Schwere deformierende Arthritis mit Ankylosen in beiden Ellenbogengelenken, in den Fingergelenken, in Knieund Fußgelenken. Schultern und Hüften weniger befallen. Starrer schmaler Brustkorb. Dyspnoe; über den Lungen diffuse trockene Rasselgeräusche, Giemen und Pfeifen. Perkussionsschall tympanitisch. Röntgenologisch bis auf Emphysem und verstärkte Hiluszeichnung, kein krankhafter Befund. Herz: Grenzen normal, auch röntgenologisch. Töne rein, jedoch außerordentlich beschleunigte Aktion, dauernd um I Io Pulse in der Minute. Bauchorgane: ohne Besonderheiten.

Therapie: Anfangs Digitalis, dann Spasmopurin 3mal täglich I Zäpfchen. Im Verlauf der Beobachtungszeit (65 Tage) tritt eine ganz wesentliche Besserung der Atembeschwerden ein. Patientin hatte anfangs gegen die rectale Applikationsweise Einwände erhoben. Es gelang mit Mühe, sie zur regelmäßigen Anwendung zu bewegen. Sie bemerkte jedoch nach kurzer Zeit eine derartig günstige Beeinflussung ihrer Dyspnoe, đaß sie die Ärzte zur Anwendung des Präparates drängte. Die Wirkung auf die Atmung trat gewöhnlich nach einer halben Stunde ein, und war bei regelmäßigen dreimaligen Gaben tagsüber eine dauernde. Versuche beim Fehlen

1) Das Präparat ist unter dem Namen "Spasmopurin" in den Handel gebracht und enthält nach Angabe dar Firma: I.3.-Dimethylxanthin 66\% $\%$, Theobromin. natr. salicyl. $33^{1 / 9} \%$. 
des Präparates, die Therapie auszusetzen, scheiterten. Es stellten sich jedesmal stärkere Atem

Wir haben nun in einer Reihe ähnlicher Fälle von Kreislaufinsuffizienz mit dyspnoischen Störungen bei älteren Personen mit dem Präparat die gleiche prompte Wirkung erzielt. Hierbei blieb die theoretisch-interessante Frage $\mathrm{zu}$ nächst offen, ob es sich bei der festgestellten Wirkung um eine direkte Beeinflussung der Bronchialmuskulatur handelte, oder ob eine sekundäre Kreislaufwirkung oder schlieBlich eine mehr allgemeine analeptische Wirkung auf das Nervensystem vorlag.

Zur Klärung des Wirkungsmechanismus sind wir dann dazu übergegangen, das Präparat bei Fällen von unkompliziertem echten Bronchialasthma Jugendlicher anzuwenden. Der Erfolg ergibt sich aus folgenden Auszügen.

Kurt L., I jjähriger Schüler, aufgenommen am 7. April 1921, Pat., der an einer chronischen. Bronchitis mitschweren asthmatischen Anfällen litt, erhielt 6 Wochen lang Spasmopurin. Die bronchitischen Erscheinungen gingen völlig zurück. Ein asthmatischer Anfall kam nach Anwendung der Therapie

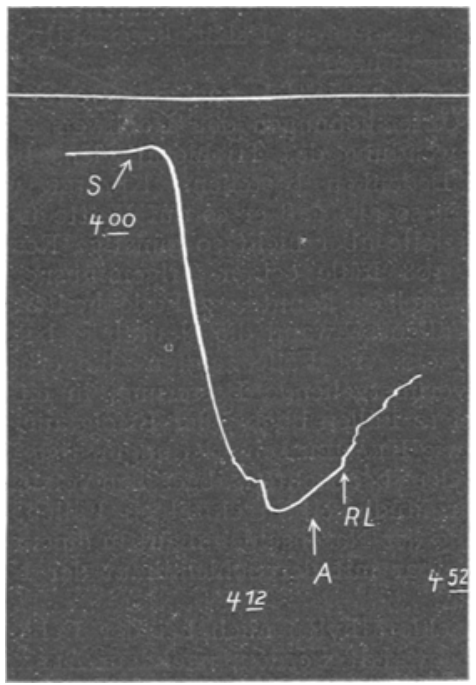

Abb. I.

Theobromin und Theophyllin (I: I000) Ppt. 4 Std. alt. uberhaupt nicht meh zur Beobachtung.

daß die eine Hauptkomponente des asthmatischen Anfalls, die spastisch kontrahierte Bronchialmuskulatur als der Angriffspunkt des Präparats $z \mathfrak{u}$ gelten habe. Aus diesem Zusammenhang ergab sich für uns ferner ein Gesichtspunkt für die Begrenzung der Purinkörpertherapie. Bei allen dyspnoischen $\mathrm{Zu}$ ständen, bei denen ein mechanischer pathogenetischer Faktor im Vordergrund steht, insbesondere bei starken entzündlichen, obliterierenden Veränderungen der Bronchialschleimhaut, bei Pleuraverwachsungen im allgemeinen bei allen Zuständen, in denen eine Dilatation der Bronchialmuskeln aus anatomischen Gründen nicht mehr möglich ist (also auch bei Bronchiektasien), darf ein Erfolg nicht erwartet werden. Es läßt sich bis $z u$ einem gewissen Grade sagen, daß der bronchodilatatorische Effekt dieser Form von Purinkörperanwendung einen diagnostischen Gradmesser darstellt für die Natur dyspnoischer Zustände, deren Ätiologie, wie eingangs erwähnt, gerade im höheren Lebensalter außerordentlich vielgestaltig ist. Es wurde schließlich versucht, die klinischen Beobachtungen durch das Experiment zu befestigen.

Es schien dies bei den spärlichen vorliegenden experimentellen Untersuchungsergebnissen um so mehr angebracht; als vergleichende Untersuchungen hinsichtlich

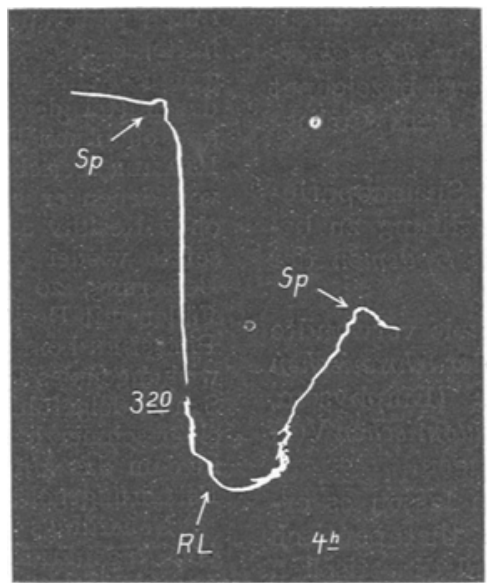

Abb. 2 .

Theobromin und Theophyllin (1: 1000) Ppt. 3 Std, alt.

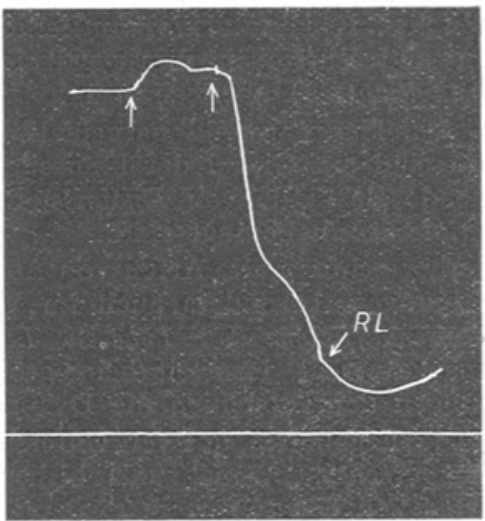

Abb. 3. Atropin (I: 1000$)$.
Noch besser ist die Einwirkung der Purinbehandlung an einem anderen Fall $\mathrm{zu}$ erkennen:

Adolf R., I 8 iähriger Hausdiener. Aufgenommen am I8. Juni 1921. In der körperlichen Entwicklung stark zurückgeblieben. Vor einem Jahr mit Schnupfen, Husten erkrankt. Starker Luftmangel, besonders nachts. In letzter Zeit starke Zunahme der Beschwerden.

Befund: Infantil aussehender junger Mann. Starker rachitischer Rosenkranz, Schmaler, wenig ausgiebig beweglicher Thorax. Perkutorischer Befund über den Lungen ohne Besonderheiten. Auskultatorisch über beiden Lungen verschärftes Atmen. Zahlreiche giemende und pfeifende Geräusche. Am Herzen hebender Spitzenstoß, Töne rein, respiratorische Arhythmie.

Diagnose: Asthma bronchiale auf endogener Grundlage. Infantilismus.

Therapie und Verlauf: Patient erhält vom 20. bis 29. Juni täglich zmal ein Suppos. Spasmopurin. Erheblicher Rückgang der Atemnot und des objektiven Befundes über den Lungen. Am 30. Juni außer Bett, beschwerdefrei. Spasmopurin abgesetzt. Am 13. Juli starker Asthmaanfall mit sofort wieder einsetzenden starken bronchitischen Erscheinungen. Auf Spasmopurin trat innerhalb eines Tages Rückgang der Erscheinungen ein. Der Patient konnte unter Anempfehlung einer Fortsetzung der Spasmopurintherapie am II. August erwerbsfähig entiassen werden.

Nach dem Ausfall dieser therapeutischen Versuche schien die Annahme, daß die günstige Beeinflussung dyspnoischer Zustände durch das Purinkörpergemisch in erster Linie einer spasmolytischen, bronchodilatatorischen Wirkung zuzuschreiber sei, wesentlich befestigt. Es durfte angenommen werden, der Wirkung der verschiedenen Purinabkömmlinge auf die Bronchialmuskulatur bisher fehlen.

Wenn auch diese experimentellen Untersuchungen, die ich im hiesigen Pharmakologischen Institut der Universität anstellen konnte, entsprechend einer weiteren Fragestellung, noch keineswegs als abgeschlossen gelten können, so bin ich doch heute schon in der Lage, ein bestimmtes Ergebnis vorwegzunehmen, da es für die hier erläuterten praktischen Fragen von Bedeutung ist. Abb. I u.2.

In Anlehnung an die TRENDELENBURGsche Methodik habe ich eine Reihe Versuche am überlebenden Bronchialmuskel des Rindes vorgenommen. Ohne auf Methodik und Einzelheiten der Versuche hier eingehen $z u$ können, seien die Ergebnisse, die bei Einwirkung des Purinkörpergemisches auf den Muskeln entstanden, an der Hand einiger Kurven wiedergegeben. Wie auf $\mathrm{Abb}$. I und 2 ohne weiteres ersichtlich, ist entsprechend der vermuteten Erweiterung der Bronchien eine ganz erhebliche Dehnung des isolierten Bronchialmuskels bei kombinierten Gaben von Theobromin und Theophyllin festzustellen. Der steile Abfall der Kurve weist zweifellos große Ahnlichkeit mit dem bei Atropinzufuhr (Abb. 3) ausgelösten Verhalten der Muskeln auf und ist in dieser Stärke von mir bisher in zahlreichen Versuchen beim gewöhnlichen Coffein. natr. salicyl. (Abb. 4) nicht zu beobachten gewesen. Abb. 3 .

Es sei allerdings ausdrücklich darauf hingewiesen, da $\beta$ die von mir verwandte Methodik eine absolute Vergleichsmöglichkeit von Wirkungen versch:edener Präparate nicht gestattet. Immorhin muß die Gleichmäßigkeit des Kurvenverlaufs bei 
Muskelstreifen, die unter durchaus nicht vergleichbaren Bedingungen gewonnen wurden, überraschen ${ }^{1}$ ). Abb. 4 .

Weiteren Versuchen

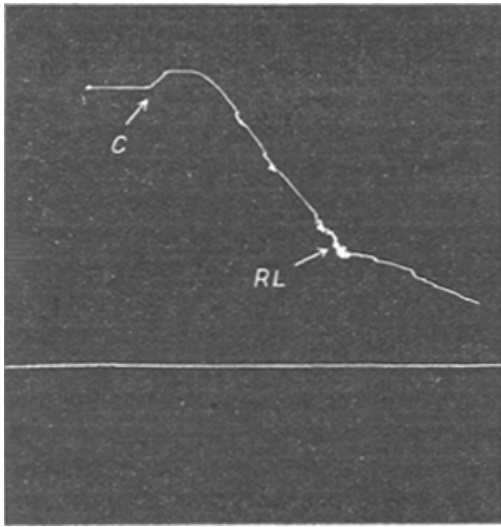
muß es überlassen bleiben, die Wirkung der verschiedenen Purinabkömmlinge möglichst quantitativ auszuwerten.

Nach allem dürfte jedoch, insbesondere unter gleichzeitiger Berücksichtigung der $k l i$ nischen Ergebnisse, auch experimentell der $\mathrm{Be}$ weis für . eine hervorragende bronchodilatotorische Wirkung des Purinkörpergemisches erbracht sein.

Abb. 4.

Coffein. natr. salicyl. (3: 1000)

Zusammenfassend ist festzustellen:

Das untersuchte Dimethylxanthingemisch zeichnet sich wie klinisch und experimentell gezeigt werden konnte, durch hervorragende Einwirkung auf den Tonus der Bronchialmuskeln aus. Es kann als krampflösendes Präparat bezeichnet werden und dürfte in dieser Hinsicht auch dem Coffein (Trimethylxanthin) überlegen sein.

2. Als Hauptanwendungsgebiet für das Spasmopuringemisch sind auf Grund der klinischen Beobachtung zu bezeichnende Zustände von Dyspnöe, die durch Spasmen der Bronchialmuskeln bedingt sind.

Hierher gehören alle Arten von Asthma, sowie verwandte Erscheinungen, besonders auch im Verlauf der chronischen Kreislaufinsuffizienz im höheren Lebensalter (Emphysem, Stauungskatarrhe), insoweit keine gröberen anatomischen Veränderungen der Bronchien die Dyspnoe bedingen.

3. Die Ergebnisse unserer Untersuchungen lassen es erwünscht erscheinen, mehr als bisher üblich therapeutisch nicht nur von den allgemein analeptischen und diuretischen Eigenschaften der Theobromine, sondern auch von ihrer bronchodilatatorischen Wirlesamkeit Gebrauch zu machen.

4. Die Harmlosigkeit der Purinkörper, im Gegensatz zu stark wirkenden Alkoloiden gestattet insbesondere bei geeigneter Darreichungsform (als Suppositorium) ihre Verwendung auch zu prophylaktischen Zwecken und über längere Zeit hinaus (evtl. bei gleichzeitiger Digitalismedikation).

\section{ERFAHRUNGEN MIT TRICHLORÄTHYLEN BEI TRIGEMINUS-NEURALGIEN.}

$$
\text { Von }
$$

\section{Dr, Kurt Magunna.}

Aus der Psychiatrischen u. Nervenklinik der Univ. Göttingen. (Direktor: Prof. Dr. Schultze.)

Vor etwa 3 Jahren wurde in der hiesigen Nervenklinik ein von SCHuLTZE veröffentlichter Fall von Encephalomyelomalazie begutachtet, die, da sie akut bei einem in einer Flugzeughalle beschäftigten Arbeiter ohne eine nachweisbare Ursache aufgetreten war, mit höchster Wahrscheinlichkeit auf eine Vergiftung mit Tetrachloräthan zurückgeführt werden konnte; Tetrachloräthan dient zur Herstellung eines Lacks, der in der Flugzeugindustrie ausgedehnte Anwendung findet. Da der Unfallverletzte zu Beginn seiner Erkrankung über Verlust des Geschmacks geklagt hatte, lag es im Hinblick auf die interessanten Mitteilungen von PLESSNER nahe, auch

1) Anmerkung bei der Korrektur: Nach Drucklegung dieser Arbeit erschien im Dezemberheft von The Journal of Pharmacology and experim. Therapeutics $192 \mathrm{r}$ (VIII, 5. S. 373): D. I. Macht und Gin-Ching Ting ,A study of antispasmodic drugs of the bronchus". Die Verff. bestätigen mittels der gleichen experimentellen Methodik wie wir, unsere Ansicht, daB den beiden Dimethylxanthinen eine stärkere bronchodilatatorische Wirkung zukommt, als dem Trimethylxanthin (Coffein). an eine Vergiftung mit dem ebenfalls als Lösungsmittel für derartige Lacke benutzten Trichloräthylen zu denken.

PLESSNER hatte r9I 5 über 4 Fälle einer gewerblichen Vergiftung mit Trichloräthylen berichtet. Die Störungen bestanden ausschließlich in einer Lähmung aller 3 Äste des sensiblen. Trigeminus. Bei den Kranken, die über die Empfindung völliger Vertaubung der Mund-, Wangen- und Nasenschleimhaut sowie der Gesichtshaut klagten, fehlte im Bereich der vorderen zwei Drittel der Zunge jede Geschmacksempfindung für süB, sauer und salzig, während bitter (N. glossopharyngeus) unterschieden wurde.

In der Aussprache zu diesem Vortrag regte OpPENHEIM an, das Trichloräthylen wegen seiner spezifischen Affinität für den sensiblen Trigeminus bei Trigeminus-Neuralgien zu versuchen.

Diesen Vorschlag griff PLessner auf. Er träufelte 3-8-20-30 -6o Tropfen auf Watte und ließ es Kranke einatmen. Er berichtete über I 2 Heilungen bei ' 7 Fällen; die übrigen 5 standen bei Veröfentlichung seiner Arbeit noch in Behandlung. Wenn auch die Zahl der beobachteten Fälle verhältnismäBig klein war, soviel schien Plessner doch erwiesen, daß die Behandlung,,wohl geeignet ist, auch schwere Fälle zu heilen, zum mindesten den Verlauf abzukürzen gegen die Zeit, die andere Behandlungsmethoden erfordern, bis sie zur Heilung führen".

Später erprobte Kramer das Mittel, nachdem JoAchimoglu durch pharmakologische Untersuchungen den Nachweis erbracht hatte, daß gegen die Anwendung des Trichloräthylens bei Trigemius-Neuralgien keine Bedenken bestehen. KRAMER wandte das Mittel gleich Plessner so an, daß er es auf Watte träufelte und einatmen lieB. Er erzielte aber nicht so günstige Resultate. KRAMER berichtete über I08 Fälle echter Trigeminusneuralgie, von denen er 58 genau verfolgen konnte. 7 Fälle heilten völlig ohne Rezidiv aus. In 5 Fällen schwand die Neuralgie, bald aber setzte wieder ein Rezidiv ein. I4. Fälle zeigten eine erhebliche Besserung, 20 eine nur vorübergehende Besserung, in einzelnen Fällen mit Rezidiven. Bei I 2 Fällen blieb jeder Erfolg aus. Auch Plessner konnte ebenso wie Kramer keinerlei ungünstige Nebenwirkungen des Trichloräthylens beobachten, abgesehen von leichtem Schwindelgefühl und einer Müdigkeit in einzelnen Fällen, sowie einer schmerzhaften Rötung der Nasenschleimhaut in einem Falle, in dem das Mittel unmittelbar mit der Schleimhaut der Nase in Berührung gekommen war.

Neuerdings ist das Trichloräthylen auch bei der Behandlung von Augenkrankheiten angewandt worden, nachdem ALEXANDER darauf hingewiesen hatte, daß es die Empfindlichkeit der Hornhaut herabsetzt oder gar aufhebt. HILDESHEIMER berichtet über eine sehr günstige Wirkung des Mittels bei Keratitis. Diese ging dank der teilweisen oder völligen Anästhesierung der Hornhaut schnell zurück, ohne daB durch diese Maßnahme das Hornhautepithel geschädigt wurde; HrLDEsheImer wie auch Kramer und Plessner fanden eine verschiedene Empfindlichkeit der einzelnen Personen gegen das Trichloräthylen.

Die eingangs erwähnte Beobachtung war der Anlaß, auch $\mathrm{n}$ der hiesigen Nervenklinik Versuche mit Trichloräthylen bei Trigeminus-Neuralgien anzustellen. Diese wurden im Juli I920 begonnen und Ende I92I beendigt.

Es standen 20 Fälle echter Trigeminus-Neuralgie zur Ver-. fügung; es handelte sich dabei nur um solche Fälle, bei denen eine besondere kausale Behandlung nicht angezeigt war. Das Mittel wurde in der Menge von Io-20 Tropfen, gelegentlich auch mehr, auf Watte geträufelt; die Kranken atmeten es mehrere Tage hintereinander, zuweilen auch $2 \mathrm{mal}$ an einem Tage und dann in kurzen Zwischenräumen ein.

Von diesen 20 Fällen wurden 5 völlig und, soweit beobachtet wurde, dauernd geheilt. 6 Fälle zeigten eine erhebliche Besserung, bei 9 Fällen versagte das Mittel völlig. Unter diesen letzten. Fällen waren 3 isolierte Neuralgien des 3 . Astes des Trigeminus, so daß es scheint, als ob der 3. Ast sich dem Mittel gegenüber refraktär verhält; jedoch müßte eine größere Untersuchungsreihe diese Vermutung bestätigen.

Was die Nebenwirkungen angeht, so trat hin und wieder für kurze Zeit ein Schwindelgefühl ein, einmal ein starkes Tränen der Augen.

Auch auf einem anderen Gebiet, bei dem das Mittel meines Wissens bisher noch nicht angewandt wurde, sah ich zuweilen günstige Erfolge; ich meine bei den hin und wieder nach einer Lumbalpunktion auftretenden Beschwerden, die sich in Kopfschmerzen und Übelkeit äußerten. 